Appl. Math. Comput 216 (2010), 3219-3227.

doi: $10.1016 /$ j.amc.2010.04.046

\title{
COMPUTER AIDED SOLUTION OF THE INVARIANCE EQUATION FOR TWO-VARIABLE STOLARSKY MEANS
}

\author{
SZABOLCS BAJÁK AND ZSOLT PÁLES
}

\begin{abstract}
We solve the so-called invariance equation in the class of two-variable Stolarsky means $\left\{S_{p, q}: p, q \in \mathbb{R}\right\}$, i.e., we find necessary and sufficient conditions on the 6 parameters $a, b, c, d, p, q$ such that the identity

$$
S_{p, q}\left(S_{a, b}(x, y), S_{c, d}(x, y)\right)=S_{p, q}(x, y) \quad\left(x, y \in \mathbb{R}_{+}\right)
$$

be valid. We recall that, for $p q(p-q) \neq 0$ and $x \neq y$, the Stolarsky mean $S_{p, q}$ is defined by

$$
S_{p, q}(x, y):=\left(\frac{q\left(x^{p}-y^{p}\right)}{p\left(x^{q}-y^{q}\right)}\right)^{\frac{1}{p-q}} .
$$

In the proof first we approximate the Stolarsky mean and we use the computer algebra system Maple V Release 9 to compute the Taylor expansion of the approximation up to 12 th order, which enables us to describe all the cases of the equality.
\end{abstract}

\section{INTRODUCTION}

Let $\mathbb{R}_{+}$denote the set of positive real numbers throughout this paper. A two-variable continuous function $M: \mathbb{R}_{+}^{2} \rightarrow \mathbb{R}_{+}$is called a mean on $\mathbb{R}_{+}$if

$$
\min (x, y) \leq M(x, y) \leq \max (x, y) \quad\left(x, y \in \mathbb{R}_{+}\right)
$$

holds. If both inequalities in (11) are strict whenever $x \neq y$, then $M$ is called a strict mean on $\mathbb{R}_{+}$.

Given three strict means $M, N, K: \mathbb{R}_{+}^{2} \rightarrow \mathbb{R}_{+}$, we say that the triple $(M, N, K)$ satisfies the invariance equation if

$$
K(M(x, y), N(x, y))=K(x, y) \quad\left(x, y \in \mathbb{R}_{+}\right)
$$

holds. If (2) holds then we say that $K$ is invariant with respect to the mean-type mapping $(M, N)$. It is well known that $K$ is uniquely determined by $M$ and $N$, and it is called the Gauss composition $K=M \otimes N$ of $M$ and $N$. For this terminology and result, see for instance the papers [15], 9].

The simplest example when the invariance equation holds is the well-known identity

$$
\sqrt{x y}=\sqrt{\frac{x+y}{2} \cdot \frac{2 x y}{x+y}} \quad\left(x, y \in \mathbb{R}_{+}\right),
$$

that is,

$$
\mathcal{G}(x, y)=\mathcal{G}(\mathcal{A}(x, y), \mathcal{H}(x, y)) \quad\left(x, y \in \mathbb{R}_{+}\right)
$$

Date: September 23, 2018.

2000 Mathematics Subject Classification. 39B10, 26E60, 94-04.

Key words and phrases. invariance equation, Gauss composition, Stolarsky mean, computer algebra.

Corresponding author: Zsolt Páles, Institute of Mathematics, University of Debrecen, 4010 Debrecen, Pf 12, Hungary.

This research was supported by the Hungarian Research Fund (OTKA) Grant Nos. NK-68040, NK81402 . 
where $\mathcal{A}, \mathcal{G}$, and $\mathcal{H}$ stand for the two-variable arithmetic, geometric, and harmonic means, respectively. Another less trivial invariance equation is the identity

$$
\mathcal{A} \otimes \mathcal{G}(x, y)=\mathcal{A} \otimes \mathcal{G}(\mathcal{A}(x, y), \mathcal{G}(x, y)) \quad\left(x, y \in \mathbb{R}_{+}\right),
$$

where $\mathcal{A} \otimes \mathcal{G}$ denotes Gauss' arithmetic-geometric mean defined by

$$
\mathcal{A} \otimes \mathcal{G}(x, y)=\left(\frac{2}{\pi} \int_{0}^{\frac{\pi}{2}} \frac{d t}{\sqrt{x^{2} \cos ^{2} t+y^{2} \sin ^{2} t}}\right)^{-1} \quad\left(x, y \in \mathbb{R}_{+}\right) .
$$

The reader is recommended to consult the book [3] for more details and history of this deep theory.

The invariance equation in more general classes of means has recently been studied extensively by many authors in various papers. The invariance of the arithmetic mean $\mathcal{A}$ (i.e., when in (2) $K$ is the arithmetic mean) with respect to two quasi-arithmetic means was first investigated by Matkowski [15] under twice continuous differentiability assumptions concerning the generating functions of the quasi-arithmetic means. These regularity assumptions were weakened step-by-step by Daróczy, Maksa, and Páles in the papers [7], [8], and finally this problem was completely solved assuming only continuity of the unknown functions involved [9]. The invariance equation involving three weighted quasi-arithmetic means was studied by Burai [5] and Jarczyk-Matkowski [14, Jarczyk [13]. The final answer (where no additional regularity assumptions are required) has been obtained in [13]. In a recent paper, we have studied the invariance of the arithmetic mean with respect to two so-called generalized quasi-arithmetic mean under four times continuous differentiability assumptions [2]. The invariance of the arithmetic mean with respect to Lagrangian means was the subject of investigation of the paper [17] by Matkowski. The invariance of the arithmetic, geometric, and harmonic means with respect to the so-called BeckenbachGini means was studied by Matkowski in [16]. Pairs of Stolarsky means for which the geometric mean is invariant were determined by Błasińska-Lesk-Głazowska-Matkowski [6]. The invariance of the arithmetic mean with respect to further means was studied by Głazowska-Jarczyk-Matkowski [12], Burai [4] and Domsta-Matkowski [10].

An important class of two variable homogeneous means are the so-called Stolarsky means (cf. Stolarsky [19]). Given two parameters $p, q \in \mathbb{R}$, the two-variable mean $S_{p, q}: \mathbb{R}_{+}^{2} \rightarrow \mathbb{R}_{+}$ is defined by the following formula

$$
S_{p, q}(x, y):= \begin{cases}\left(\frac{q\left(x^{p}-y^{p}\right)}{p\left(x^{q}-y^{q}\right)}\right)^{\frac{1}{p-q}}, & \text { if }(p-q) p q \neq 0, x \neq y \\ \exp \left(-\frac{1}{p}+\frac{x^{p} \log x-y^{p} \log y}{x^{p}-y^{p}}\right), & \text { if } p=q \neq 0, x \neq y, \\ \left(\frac{x^{p}-y^{p}}{p(\log x-\log y)}\right)^{\frac{1}{p}}, & \text { if } p \neq 0, q=0, x \neq y, \\ \left(\frac{q(\log x-\log y)}{x^{q}-y^{q}}\right)^{-\frac{1}{q}}, & \text { if } p=0, q \neq 0, x \neq y, \\ \sqrt{x y}, & \text { if } p=q=0, \\ x, & \text { if } x=y,\end{cases}
$$

for every $x, y \in \mathbb{R}_{+}$.

It can easily be seen that the power (or Hölder) mean of exponent $p$ can be obtained as $S_{2 p, p}$. In particular, $S_{2,1}, S_{0,0}$ and $S_{-2,-1}$ are the arithmetic, geometric, and harmonic means, respectively. Moreover, it follows by a simple computation that $S_{p, q}$ is equal to the geometric mean whenever $p+q=0$. One can also check that the Stolarsky means are 
symmetric with respect to their variables and their parameters as well, i.e., $S_{p, q}(x, y)=$ $S_{p, q}(y, x)=S_{q, p}(x, y)$ for all $x, y \in \mathbb{R}_{+}$and $p, q \in \mathbb{R}$.

The aim of this paper is to solve the invariance equation in the class of Stolarsky means, i.e., to solve (2) when each of the means $M, N$ and $K$ is a Stolarsky mean. More precisely, we want to describe the set of all 6-tuples $(a, b, c, d, p, q)$ such that the identity

$$
S_{p, q}\left(S_{a, b}(x, y), S_{c, d}(x, y)\right)=S_{p, q}(x, y) \quad\left(x, y \in \mathbb{R}_{+}\right)
$$

holds. The analogous problem concerning the so-called Gini means, i.e., the description of the set of all 6-tuples $(a, b, c, d, p, q)$ such that

$$
G_{p, q}\left(G_{a, b}(x, y), G_{c, d}(x, y)\right)=G_{p, q}(x, y) \quad\left(x, y \in \mathbb{R}_{+}\right)
$$

be valid, has recently been solved in the paper [1] by the authors. Recall that given two parameters $p, q \in \mathbb{R}$, the two-variable mean $G_{p, q}: \mathbb{R}_{+}^{2} \rightarrow \mathbb{R}_{+}$is defined by the following formula (cf. Gini [11]):

$$
G_{p, q}(x, y)= \begin{cases}\left(\frac{x^{p}+y^{p}}{x^{q}+y^{q}}\right)^{\frac{1}{p-q}} & \text { for } p \neq q, \\ \exp \left(\frac{x^{p} \log x+y^{p} \log y}{x^{p}+y^{p}}\right) & \text { for } p=q,\end{cases}
$$

for $x, y \in \mathbb{R}_{+}$. The class of Gini means is also a generalization of the class of power means, since taking $q=0$, we immediately get the power (or Hölder) mean of exponent $p$. The main result of the paper [1] is contained in the following theorem:

Theorem G. Let $a, b, c, d, p, q \in \mathbb{R}$. Then the invariance equation (4) is satisfied if and only if one of the following possibilities hold:

(i) $a+b=c+d=p+q=0$, i.e., all the three means are equal to the geometric mean,

(ii) $\{a, b\}=\{c, d\}=\{p, q\}$, i.e., all the three means are equal to each other,

(iii) $\{a, b\}=\{-c,-d\}$ and $p+q=0$, i.e., $G_{p, q}$ is the geometric mean and $G_{a, b}=G_{-c,-d}$,

(iv) there exist $u, v \in \mathbb{R}$ such that $\{a, b\}=\{u+v, v\},\{c, d\}=\{u-v,-v\}$, and $\{p, q\}=$ $\{u, 0\}$ (in this case, $G_{p, q}$ is a power mean),

(v) there exists $w \in \mathbb{R}$ such that $\{a, b\}=\{3 w, w\}, c+d=0$, and $\{p, q\}=\{2 w, 0\}$ (in this case, $G_{p, q}$ is a power mean and $G_{c, d}$ is the geometric mean),

(vi) there exists $w \in \mathbb{R}$ such that $a+b=0,\{c, d\}=\{3 w, w\}$, and $\{p, q\}=\{2 w, 0\}$ (in this case, $G_{p, q}$ is a power mean and $G_{a, b}$ is the geometric mean).

As an obvious consequence, we obtain the following solution for the so-called MatkowskiSutô equation, i.e., when $G_{p, q}$ is equal to the arithmetic mean in the invariance equation (4), which happens exactly when $\{p, q\}=\{1,0\}$.

Corollary G. Let $a, b, c, d \in \mathbb{R}$. Then the Matkowski-Sutô equation

$$
G_{a, b}(x, y)+G_{c, d}(x, y)=x+y \quad\left(x, y \in \mathbb{R}_{+}\right)
$$

is satisfied if and only if one of the following possibilities hold:

(i) $\{a, b\}=\{c, d\}=\{1,0\}$, i.e., the two means are equal to the arithmetic mean,

(ii) there exists $v \in \mathbb{R}$ such that $\{a, b\}=\{1+v, v\},\{c, d\}=\{1-v,-v\}$,

(iii) $\{a, b\}=\left\{\frac{3}{2}, \frac{1}{2}\right\}$ and $c+d=0$ (in this case, $G_{c, d}$ is the geometric mean),

(iv) $a+b=0$ and $\{c, d\}=\left\{\frac{3}{2}, \frac{1}{2}\right\}$ (in this case, $G_{a, b}$ is the geometric mean).

The approach followed in [1] heavily used the Maple computer-algebra system to perform the tedious computations of the various partial derivatives of Gini means up to 12th order.

The main result of this paper completely solves the invariance equation in the class of Stolarsky means. 
Theorem S. Let $a, b, c, d, p, q \in \mathbb{R}$. Then the invariance equation (3) is valid if and only if one of the following possibilities holds:

(i) $a+b=c+d=p+q=0$, i.e., all the three means are equal to the geometric mean,

(ii) $\{a, b\}=\{c, d\}=\{p, q\}$, i.e., all the three means are equal to each other,

(iii) $\{a, b\}=\{-c,-d\}$ and $p+q=0$, i.e., $S_{p, q}$ is the geometric mean and $S_{a, b}=S_{-c,-d}$.

As a consequence, we obtain the following solution for the Matkowski-Sutô equation, i.e., when $S_{p, q}$ is equal to the arithmetic mean in the invariance equation (3), which happens exactly when $\{p, q\}=\{2,1\}$.

Corollary S. Let $a, b, c, d \in \mathbb{R}$. Then the Matkowski-Sutô equation

$$
S_{a, b}(x, y)+S_{c, d}(x, y)=x+y \quad\left(x, y \in \mathbb{R}_{+}\right)
$$

holds if and only if $\{a, b\}=\{c, d\}=\{2,1\}$, i.e., both means are equal to the arithmetic mean.

It is interesting to observe here that the parameter sets when (4) holds is much bigger then the corresponding set for (3).

In the proof of the above results first we construct an approximation of the Stolarsky means. Then we use the computer algebra system Maple V Release 9 to compute the Taylor expansion of a function (which is in terms of the approximated means) up to 12th order. Finally, this enables us to describe all the cases of the equality.

\section{The PRoOF of TheOREM S}

First we recall the characterization of the equality of two variable Stolarsky means.

Lemma 1. (Cf. [18]) Let $a, b, c, d \in \mathbb{R}$. Then the identity

$$
S_{a, b}(x, y)=S_{c, d}(x, y) \quad\left(x, y \in \mathbb{R}_{+}\right)
$$

holds if and only if one of the following possibilities is valid:

(i) $a+b=c+d=0$ and, in this case, the two means are equal to the geometric mean,

(ii) $\{a, b\}=\{c, d\}$.

The calculations of the higher-order partial derivatives of Stolarsky means at the point $(x, y)=(1,1)$ is too complicated (even for computer-algebra systems) because the main expression that defines these means is singular at diagonal points. Therefore, to simplify these computations, we will approximate the Stolarsky mean up to a sufficiently high order. First, we express the Stolarsky mean in another form.

Lemma 2. For $p, q \in \mathbb{R}$ and $x, y \in \mathbb{R}_{+}$, the Stolarsky mean $S_{p, q}(x, y)$ can be rewritten as (6)

$$
S_{p, q}(x, y):= \begin{cases}\left(\frac{L(p \log x, p \log y)}{L(q \log x, q \log y)}\right)^{\frac{1}{p-q}} & \text { if } p \neq q, \\ \exp \left(\frac{\partial_{1} L(p \log x, p \log y) \log x+\partial_{2} L(p \log x, p \log y) \log y}{L(p \log x, p \log y)}\right) & \text { if } p=q,\end{cases}
$$

where the function $L: \mathbb{R}^{2} \rightarrow \mathbb{R}$ is defined by

$$
L(u, v):=\sum_{n=1}^{\infty} \frac{u^{n-1}+u^{n-2} v+\cdots+u v^{n-2}+v^{n-1}}{n !} .
$$


Proof. Utilizing the Taylor series expansion and substituting $u:=\log x$ and $v:=\log y$, we have, for $p \neq 0$ and $x \neq y$,

$$
\begin{aligned}
\frac{x^{p}-y^{p}}{p(\log x-\log y)} & =\frac{\mathrm{e}^{p u}-\mathrm{e}^{p v}}{p(u-v)}=\frac{1}{p(u-v)}\left(1+\frac{p u}{1 !}+\frac{(p u)^{2}}{2 !}+\cdots-1-\frac{p v}{1 !}-\frac{(p v)^{2}}{2 !}-\cdots\right) \\
& =\frac{1}{u-v}\left(\frac{u-v}{1 !}+\frac{p\left(u^{2}-v^{2}\right)}{2 !}+\frac{p^{2}\left(u^{3}-v^{3}\right)}{3 !}+\cdots\right) \\
& =1+\frac{p(u+v)}{2 !}+\frac{p^{2}\left(u^{2}+u v+v^{2}\right)}{3 !}+\cdots=L(p u, p v) .
\end{aligned}
$$

Similarly, we can get that $L(p u, p u)=\mathrm{e}^{p u}$, hence

$$
L(p \log x, p \log y):= \begin{cases}\frac{x^{p}-y^{p}}{p(\log x-\log y)}, & \text { if } p \neq 0, x \neq y \\ x^{p}, & \text { if } x=y \\ 1, & \text { if } p=0\end{cases}
$$

Using the above identity and the definition of the Stolarsky means, equality (6) follows immediately for $p \neq q$. The formula for the case $p=q$ is derived by taking the limit $q \rightarrow p$.

In order to obtain high-order approximation of Stolarsky means, for $k \in \mathbb{N}$, define

$$
L_{k}(u, v):=\sum_{n=1}^{k} \frac{u^{n-1}+u^{n-2} v+\cdots+u v^{n-2}+v^{n-1}}{n !} \quad(u, v \in \mathbb{R}),
$$

and, for $x, y \in \mathbb{R}_{+}, p, q \in \mathbb{R}$,

$$
S_{p, q}^{k}(x, y):= \begin{cases}\left(\frac{L_{k}(p \log x, p \log y)}{L_{k}(q \log x, q \log y)}\right)^{\frac{1}{p-q}} & \text { if } p \neq q, \\ \exp \left(\frac{\partial_{1} L_{k}(p \log x, p \log y) \log x+\partial_{2} L_{k}(p \log x, p \log y) \log y}{L_{k}(p \log x, p \log y)}\right) & \text { if } p=q .\end{cases}
$$

Lemma 3. For $p, q \in \mathbb{R}, k \in \mathbb{N}$,

$$
\partial_{1}^{i} \partial_{2}^{j} S_{p, q}(1,1)=\partial_{1}^{i} \partial_{2}^{j} S_{p, q}^{k}(1,1)
$$

if $i \geq 1, j \geq 1$ and $i+j \leq k$.

Proof. Observe that, for $\alpha \geq 0, \beta \geq 0$ with $\alpha+\beta \leq k$, we have

$$
\partial_{1}^{\alpha} \partial_{2}^{\beta} L(0,0)=\partial_{1}^{\alpha} \partial_{2}^{\beta} L_{k}(0,0) .
$$

In the case $p \neq q$, by applying the elementary rules of differentiation and the identities (6) and (9)), we get that the formulae for the partial derivatives $\partial_{1}^{i} \partial_{2}^{j} S_{p, q}(1,1)$ and $\partial_{1}^{i} \partial_{2}^{j} S_{p, q}^{k}(1,1)$ are the same expressions of all the partial derivatives $\partial_{1}^{\alpha} \partial_{2}^{\beta} L(0,0)$ and $\partial_{1}^{\alpha} \partial_{2}^{\beta} L_{k}(0,0)$, respectively, where $0 \leq \alpha \leq i$ and $0 \leq \beta \leq j$. Hence, equality (10) follows for $p \neq q$. In the case $p=q$, the identity is derived by taking the limit $q \rightarrow p$.

To make the calculations even more simple, we define the function $E: \mathbb{R}^{4} \rightarrow \mathbb{R}$, by

$$
E(p, q, u, v):=\log \left(S_{p, q}\left(e^{u}, e^{v}\right)\right)= \begin{cases}\frac{\log L(p u, p v)-\log L(q u, q v)}{p-q} & \text { if } p \neq q, \\ \frac{\partial_{1} L(p u, p v) u+\partial_{2} L(p u, p v) v}{L(p u, p v)} & \text { if } p=q .\end{cases}
$$

The invariance equation (3) can be rewritten in the form

$$
E(p, q, E(a, b, u, v), E(c, d, u, v))=E(p, q, u, v) \quad(u, v \in \mathbb{R}),
$$


hence

$$
F(x):=E(p, q, E(a, b, x,-x), E(c, d, x,-x))-E(p, q, x,-x)=0 \quad(x \in \mathbb{R}) .
$$

Thus, the derivatives $F^{(m)}$ vanish at $x=0$ for all $m \geq 0, m \in \mathbb{Z}$. By the symmetry of Stolarsky means, $F$ is an even function. Therefore, $F^{(2 m+1)}(0)=0$ holds automatically. In order to obtain the necessity of the conditions of the theorem, we will need to investigate the equalities

$$
F^{(2 m)}(0)=0, \quad(1 \leq m \leq 6) .
$$

To compute these derivatives, define the function $F_{k}: \mathbb{R} \rightarrow \mathbb{R}, k \in \mathbb{N}$, as

$$
F_{k}(x):=E_{k}\left(p, q, E_{k}(a, b, x,-x), E_{k}(c, d, x,-x)\right)-E_{k}(p, q, x,-x),
$$

where

$$
E_{k}(p, q, u, v):=\log \left(S_{p, q}^{k}\left(e^{u}, e^{v}\right)\right)= \begin{cases}\frac{\log L_{k}(p u, p v)-\log L_{k}(q u, q v)}{p-q} & \text { if } p \neq q, \\ \frac{\partial_{1} L_{k}(p u, p v) u+\partial_{2} L_{k}(p u, p v) v}{L_{k}(p u, p v)} & \text { if } p=q .\end{cases}
$$

It follows from Lemma 3 that the derivatives of the functions $E_{k}$ and $E$ at $(p, q, 0,0)$, and hence the derivatives of $F_{k}$ and $F$ up to the order $k$ at the point $x=0$ coincide. Therefore, to analyze the equations (11), it is sufficient to consider the identities

$$
F_{k}^{(2 m)}(0)=0, \quad(1 \leq m \leq 6),
$$

where $2 m \leq k$. Thus in the proof of the theorem, we compute the Taylor expansion of the appropriate $F_{k}$ at $x=0$, hence we can obtain conditions for the unknown parameters $a, b, c, d, p, q$.

The proof of Theorem $S$. Assume that (3) holds. In the syntax of the Maple language, we define the functions $L_{k}, E_{k}$ and $F_{k}$ in the following way:

$$
\begin{aligned}
& \mathrm{L}:=(\mathrm{u}, \mathrm{v}, \mathrm{k})->\operatorname{add}\left((1 / \mathrm{n} !) * \operatorname{add}\left(\mathrm{u}^{\wedge}(\mathrm{n}-\mathrm{i}) * \mathrm{v}^{\wedge}(\mathrm{i}-1), \mathrm{i}=1 \ldots \mathrm{n}\right), \mathrm{n}=1 \ldots \mathrm{k}\right) ; \\
& \mathrm{E}:=(\mathrm{p}, \mathrm{q}, \mathrm{u}, \mathrm{v}, \mathrm{k})->(1 /(\mathrm{p}-\mathrm{q})) *(\ln (\mathrm{L}(\mathrm{pu}, \mathrm{pv}, \mathrm{k}))-\ln (\mathrm{L}(\mathrm{qu}, \mathrm{qv}, \mathrm{k}))) ; \\
& \mathrm{F}:=(\mathrm{x}, \mathrm{k})->\mathrm{E}(\mathrm{p}, \mathrm{q}, \mathrm{E}(\mathrm{a}, \mathrm{b}, \mathrm{x},-\mathrm{x}, \mathrm{k}), \mathrm{E}(\mathrm{c}, \mathrm{d}, \mathrm{x},-\mathrm{x}, \mathrm{k}), \mathrm{k})-\mathrm{E}(\mathrm{p}, \mathrm{q}, \mathrm{x},-\mathrm{x}, \mathrm{k}) ;
\end{aligned}
$$

This produces the following output:

$$
\begin{aligned}
L & :=(u, v, k) \rightarrow \operatorname{add}\left(\frac{\operatorname{add}\left(u^{n-i} v^{i-1}, i=1 \ldots n\right)}{n !}, n=1 \ldots k\right), \\
E & :=(p, q, u, v, k) \rightarrow \frac{\log (L(p u, p v, k))-\log (L(q u, q v, k))}{p-q}, \\
F & :=(x, k) \rightarrow E(p, q, E(a, b, x,-x, k), E(c, d, x,-x, k), k)-E(p, q, x,-x, k) .
\end{aligned}
$$

First we evaluate the second-order Taylor coefficient $C_{2}$ of $F$ at $x=0$ by replacing $F$ by $F_{3}$ :

$>C[2]:=\operatorname{simplify}(\operatorname{coeftayl}(\mathrm{F}(\mathrm{x}, 3), \mathrm{x}=0,2))$;

$$
C_{2}=\frac{1}{12} d-\frac{1}{6} q+\frac{1}{12} p-\frac{1}{6} q+\frac{1}{12} a+\frac{1}{12} c .
$$

(Note that the Maple-definition of the function $E$ is valid only if $(p-q)(a-b)(c-d) \neq 0$, however, the Taylor coefficient $C_{2}$ and also the subsequent ones, are correct also in the singular case $(p-q)(a-b)(c-d)=0$.)

By the invariance equation $C_{2}=0$, therefore

$$
\frac{a+b+c+d}{4}=\frac{p+q}{2}
$$


In order to simplify the evaluation of the higher-order Taylor coefficients, we introduce the notations

$$
\begin{aligned}
w & :=\frac{a+b+c+d}{4}=\frac{p+q}{2}, \\
v & :=\frac{a+b-(c+d)}{4}, \\
t & :=\left(\frac{p-q}{2}\right)^{2} \\
r & :=\frac{(a-b)^{2}+(c-d)^{2}}{8}, \\
s & :=\frac{(a-b)^{2}-(c-d)^{2}}{8} .
\end{aligned}
$$

(In the definition of $w$ we utilized the condition $C_{2}=0$.) Then we can express the parameters $a, b, c, d, p, q$ in the following form:

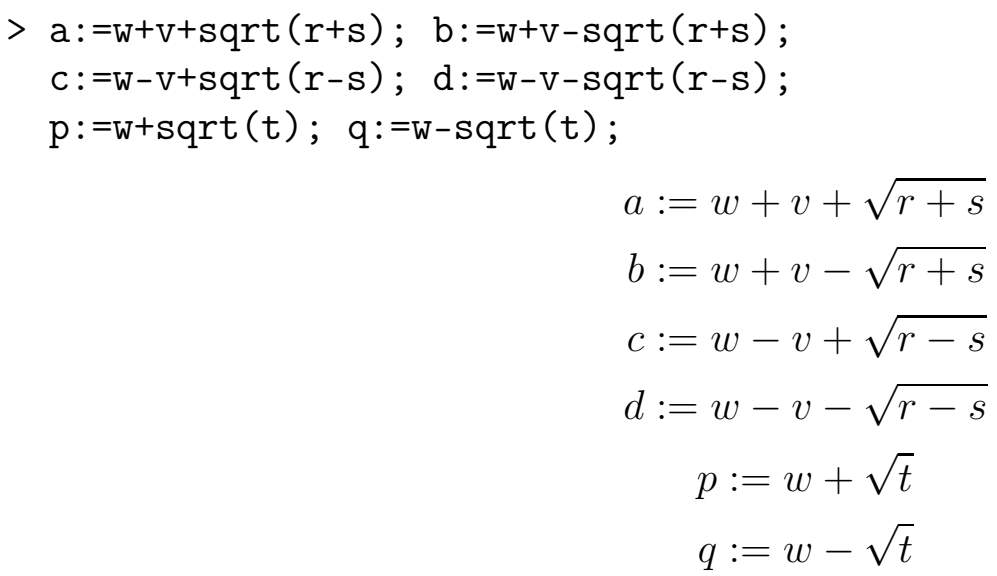

Now we evaluate the 4 th order Taylor coefficient $C_{4}$ of $F$ (i.e., of $F_{5}$ ) at $x=0$ by inputting:

$>C[4]:=\operatorname{simplify}(\operatorname{coeftayl}(F(x, 5), x=0,4))$;

$$
C_{4}:=-\frac{1}{45} v s-\frac{4}{135} w v^{2}+\frac{1}{45} w t-\frac{1}{45} w r
$$

The condition $C_{4}=0$ yields that $w t=w r+v s+\frac{4}{3} w v^{2}$.

If $w=0$, then $p+q=0$, which means that $S_{p, q}$ is the geometric mean. Therefore, the invariance equation can be written as

$$
S_{a, b}(x, y) S_{c, d}(x, y)=x y \quad\left(x, y \in \mathbb{R}_{+}\right) .
$$

This results

$$
S_{a, b}(x, y)=\frac{1}{S_{c, d}(1 / x, 1 / y)}=S_{-c,-d}(x, y) \quad\left(x, y \in \mathbb{R}_{+}\right) .
$$

Using Lemma 1, this identity yields that either $a+b=c+d=0$ or $\{a, b\}=\{-c,-d\}$ must hold. In this case we get that one of the conditions (i) or (iii) of our theorem is valid. Conversely, if conditions (i) or (iii) hold then (3) can easily be seen.

In the rest of the proof, we may assume that $w$ is not zero. Then, from condition $C_{4}=0$, we can express $t$ in terms of $w, v, r, s$ :

$>\mathrm{t}:=\mathrm{r}+\mathrm{v} * \mathrm{~s} / \mathrm{w}+(4 / 3) * \mathrm{v}^{\wedge} 2$

$$
t:=r+\frac{v s}{w}+\frac{4}{3} v^{2}
$$

Next, we evaluate the 6 th order Taylor coefficient $C_{6}$ of $F$ (i.e., of $F_{7}$ ) at $x=0$ : 
$>C[6]:=\operatorname{simplify}(\operatorname{coeftayl}(F(x, 7), x=0,6))$;

We get that

$$
C_{6}:=\frac{2\left(9 w^{2} s^{2}+8 w^{2} v^{4}+45 w^{2} r v^{2}+39 w^{3} s v+6 w v^{3} s-13 w^{4} v^{2}-9 v^{2} s^{2}\right)}{8505 w}
$$

If $v=0$, then $C_{6}=0$ implies that $s=0$. Hence, from (14) it follows that $t=r$ and we get that $a=c=p$ and $b=d=q$, i.e., condition (ii) of our theorem holds. Conversely, if condition (ii) holds then the invariance equation (3) is trivially valid.

In the rest of the proof, we may assume that $v$ is also not zero. Observe that the 6th order coefficient $C_{6}$ does not involve higher-order powers of $r$. Therefore, the equation $C_{6}=0$ can be solved for $r$. Temporarily, we denote this solution by $R$ :

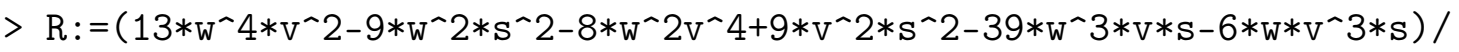

$\left(45 * \mathrm{w}^{\wedge} 2 * \mathrm{v}^{\wedge} 2\right)$;

$$
R:=\frac{13 w^{4} v^{2}-9 w^{2} s^{2}-8 w^{2} v^{4}+9 v^{2} s^{2}-39 w^{3} v s-6 w v^{3} s}{45 w^{2} v^{2}}
$$

Finally, we evaluate the 13th order Taylor polynomial of $F_{13}$ at $x=0$ (the Maple output is suppressed by putting : instead of ; to the end of the Maple command, for the sake of brevity), then we extract the 8 th, 10 th and 12 th order Taylor coefficients, denoted by $C_{8}$, $C_{10}$ and $C_{12}$, respectively, and replace $r$ by $R$ by inputting:

$>\mathrm{T}:=$ simplify (taylor $(\mathrm{F}(\mathrm{x}, 13), \mathrm{x}=0,13))$ :

for $i$ from 8 to 12 by 2

do $C[i]:=\operatorname{simplify}(\operatorname{subs}(r=R, \operatorname{simplify}(\operatorname{coeff}(T, x, i)))$, factor $)$ od;

$$
\begin{aligned}
C_{8}:= & \frac{1}{9568125 w^{3} v^{2}}\left(4347 w^{5} v s^{3}-3616 w^{8} v^{4}+1242 w^{2} v^{6} s^{2}+16137 w^{6} v^{2} s^{2}+224 w^{4} v^{8}\right. \\
& -4644 w^{3} v^{3} s^{3}+756 w^{3} v^{7} s+81 w^{4} s^{4}+11976 w^{7} v^{3} s+4992 w^{6} v^{6}-162 w^{2} v^{2} s^{4} \\
& \left.-17379 w^{4} v^{4} s^{2}-12732 w^{5} v^{5} s+297 w v^{5} s^{3}+81 v^{4} s^{4}\right)
\end{aligned}
$$

$$
\begin{aligned}
& C_{10}:=\frac{2}{2841733125 w^{5} v^{4}}\left(141632 w^{10} v^{8}-2187 w^{2} v^{4} s^{6}-58806 w^{3} v^{5} s^{5}+107406 w^{5} v^{3} s^{5}\right. \\
& -338432 w^{12} v^{6}++34928 w^{6} v^{12}-523908 w^{9} v^{3} s^{3}+2187 w^{4} v^{2} s^{6}+126459 w^{4} v^{10} s^{2} \\
& +316272 w^{8} v^{10}+143541 w^{8} v^{6} s^{2}+92508 w^{5} v^{11} s+24948 w^{2} v^{8} s^{4}+1319016 w^{11} v^{5} s \\
& -847044 w^{9} v^{7} s+927324 w^{10} v^{4} s^{2}-1197324 w^{6} v^{8} s^{2}-564480 w^{7} v^{9} s-729 w^{6} s^{6} \\
& +55026 w^{3} v^{9} s^{3}-794610 w^{5} v^{7} s^{3}+672786 w^{6} v^{4} s^{4}+3402 w v^{7} s^{5}-52002 w^{7} v s^{5} \\
& \left.-313065 w^{4} v^{6} s^{4}+1263492 w^{7} v^{5} s^{3}-384669 w^{8} v^{2} s^{4}+729 v^{6} s^{6}\right)
\end{aligned}
$$




$$
\begin{aligned}
C_{12}:= & \frac{2}{872767286015625 w^{7} v^{6}}\left(54403812 w^{4} v^{4} s^{8}-36269208 w^{6} v^{2} s^{8}-2472351012 w^{7} v^{3} s^{7}\right. \\
& -36269208 w^{2} v^{6} s^{8}-6728400999 w^{4} v^{8} s^{6}-23518313469 w^{8} v^{4} s^{6}+28808296272 w^{10} v^{14} \\
& -43502512128 w^{16} v^{8}-32563674368 w^{14} v^{10}+55532868672 w^{12} v^{12}+4082581552 w^{8} v^{16} \\
& +51381378 w v^{9} s^{7}+1616450580 w^{3} v^{11} s^{5}+66139190820 w^{7} v^{7} s^{5}+5633126127 w^{4} v^{12} s^{4} \\
& -27686800350 w^{5} v^{9} s^{5}+23171026860 w^{11} v^{3} s^{5}-198268186320 w^{13} v^{5} s^{3}+9067302 w^{8} s^{8} \\
& -50400222003 w^{12} v^{4} s^{4}+8922828051 w^{10} v^{2} s^{6}+527871816 w^{2} v^{10} s^{6}+2575113768 w^{5} v^{5} s^{7} \\
& +33853773582 w^{10} v^{6} s^{4}+8610065640 w^{5} v^{13} s^{3}+78734957472 w^{8} v^{8} s^{4}+806989878 w^{9} v s^{7} \\
& -56327456871 w^{8} v^{12} s^{2}-297179050266 w^{10} v^{10} s^{2}-14684723544 w^{13} v^{9} s \\
& +203758408416 w^{15} v^{7} s-195782704164 w^{11} v^{11} s-67821635178 w^{6} v^{10} s^{4} \\
& +10150547496 w^{7} v^{15} s+13660568109 w^{6} v^{14} s^{2}-84844566450 w^{7} v^{11} s^{3} \\
& +337457994084 w^{12} v^{8} s^{2}+2387944944 w^{14} v^{6} s^{2}-3441528204 w^{9} v^{13} s \\
& -67735201590 w^{9} v^{9} s^{3}+342237888720 w^{11} v^{7} s^{3}-961134012 w^{3} v^{7} s^{7} \\
& \left.+20796014601 w^{6} v^{6} s^{6}-63239867910 w^{9} v^{5} s^{5}+9067302 v^{8} s^{8}\right)
\end{aligned}
$$

The Taylor coefficients $C_{8}, C_{10}$, and $C_{12}$ are of the form

$$
C_{8}=\frac{1}{9568125 w^{3} v^{2}} P_{8}, \quad C_{10}=\frac{2}{2841733125 w^{5} v^{4}} P_{10}, \quad C_{12}=\frac{2}{872767286015625 w^{7} v^{6}} P_{12},
$$

where $P_{8}, P_{10}$, and $P_{12}$ are polynomials of the variables $v, w, s$. They can be obtained by the following Maple commands (whose output is suppressed):

$>\quad \mathrm{P}[8]:=\mathrm{op}(2, \mathrm{C}[8]): \mathrm{P}[10]:=\mathrm{op}(2, \mathrm{C}[10]): \mathrm{P}[12]:=\mathrm{op}(2, \mathrm{C}[12]):$

The equalities $C_{8}=C_{10}=C_{12}=0$ imply that $P_{8}=P_{10}=P_{12}=0$.

The variable $s$ is a common root of the polynomials $P_{8}$ and $P_{10}$. Therefore the resultant $R_{8,10}$ of these two polynomials (with respect to $s$ ) is zero:

$>\mathrm{R}[8,10]:=$ factor $($ resultant $(\mathrm{P}[8], \mathrm{P}[10], \mathrm{s}))$;

$$
\begin{aligned}
& R_{8,10}:=28242953648100000000 w^{24} v^{24}(v-w)^{8}(v+w)^{8}\left(395726752304 v^{32}-28019198519832 w^{2} v^{30}\right. \\
& +1192972799035666 w^{4} v^{28}-36617671790074251 w^{6} v^{26}+601554420387156651 w^{8} v^{24} \\
& -3652037976710860175 w^{10} v^{22}-1101310194408221307 w^{12} v^{20}+62048533824813847173 w^{14} v^{18} \\
& -175575191501013599783 w^{16} v^{16}+52614376847529172973 w^{18} v^{14}+435211540238087039223 w^{20} v^{12} \\
& -793895884964266327270 w^{22} v^{10}-773252618095825970136 w^{24} v^{8}-492199682627262911866 w^{26} v^{6} \\
& \left.+183522699320559043726 w^{28} v^{4}-43030934088053846752 w^{30} v^{2}+912066926976343384 w^{32}\right)
\end{aligned}
$$

The resultant is zero if either $v w(v-w)(v+w)=0$ holds or $v$ and $w$ are solutions of a homogeneous two variable polynomial equation of degree 32. Writing $w$ in the form

$>\mathrm{w}:=\mathrm{Z} * \mathrm{v}$

$$
w:=z v
$$

we get that $z$ is a root of a 32 nd degree polynomial $P_{8,10}$, where:

$>\mathrm{P}[8,10]:=\operatorname{simplify}\left(\mathrm{op}(4, \mathrm{R}[8,10]) / \mathrm{V}^{\wedge} 32\right)$; 


$$
\begin{aligned}
P_{8,10} & :=395726752304-28019198519832 z^{2}+1192972799035666 z^{4}-36617671790074251 z^{6} \\
& +601554420387156651 z^{8}-3652037976710860175 z^{10}-1101310194408221307 z^{12} \\
& +62048533824813847173 z^{14}-175575191501013599783 z^{16}+52614376847529172973 z^{18} \\
& -435211540238087039223 z^{20}-793895884964266327270 z^{22}+773252618095825970136 z^{24} \\
& -492199682627262911866 z^{26}+183522699320559043726 z^{28}-43030934088053846752 z^{30} \\
& +912066926976343384 z^{32} .
\end{aligned}
$$

The variable $s$ is also a common root of the two polynomials $P_{8}$ and $P_{12}$. Therefore the resultant $R_{8,12}$ of these polynomials (with respect to $s$ ) is again zero:

$>\mathrm{R}[8,12]:=$ factor $($ resultant $(\mathrm{P}[8], \mathrm{P}[12], \mathrm{s}))$ :

We now get that $v$ and $w$ are solutions of a homogeneous two variable polynomial of degree 44 , whence we get that $z$ is a root of the 44 th degree polynomial $P_{8,12}$, where:

$>\mathrm{P}[8,12]:=\mathrm{op}(4, \mathrm{R}[8,12])$;

Now computing the resultant of the two polynomials $P_{8,10}$ and $P_{8,12}$ by

$>\mathrm{Q}:=$ resultant $(\mathrm{P}[8,10], \mathrm{P}[8,12], \mathrm{z}):$

it follows that $Q$ is a (huge) nonzero number, hence $P_{8,10}$ and $P_{8,12}$ cannot have a common root. This proves that $R_{8,10}$ and $R_{8,12}$ can be simultaneously zero if and only if $v w(v-$ $w)(v+w)=0$ holds. Hence $C_{8}=C_{10}=C_{12}=0$ can hold only in this case.

Since we have $v w \neq 0$, hence $(v-w)(v+w)=0$ must hold, i.e, $v= \pm w$. Thus, from

(15) and (14), we get that $r=\frac{w^{2}}{9} \mp s$ and $t=\frac{13}{9} w^{2}$, respectively.

In the case when $v=w$, the equations in (13) yield that

$$
\text { (16) } c=-d, \quad a=\frac{7}{3} w, \quad b=\frac{5}{3} w, \quad p=\left(1+\frac{\sqrt{13}}{3}\right) w, \quad q=\left(1-\frac{\sqrt{13}}{3}\right) w .
$$

The first equality yields that $S_{c, d}$ is the geometric mean. Hence, we may assume that $c=-d=1$. To simplify the computations, we can also assume that $w=3$. We show that these parameters are not solutions of the invariance equation. For $k \in \mathbb{N}$, we now have that

$F_{k}(x):=E_{k}\left(3+\sqrt{13}, 3-\sqrt{13}, E_{k}(7,5, x,-x), E_{k}(1,-1, x,-x)\right)-E_{k}(3+\sqrt{13}, 3-\sqrt{13}, x,-x)$.

In Maple, we input

$>\mathrm{F}:=(\mathrm{x}, \mathrm{k})->\mathrm{E}(3+\operatorname{sqrt}(13), 3-\operatorname{sqrt}(13), \mathrm{E}(7,5, \mathrm{x},-\mathrm{x}, \mathrm{k}), \mathrm{E}(1,-1, \mathrm{x},-\mathrm{x}, \mathrm{k}), \mathrm{k})-$

$\mathrm{E}(3+\operatorname{sqrt}(13), 3-\operatorname{sqrt}(13), \mathrm{x},-\mathrm{x}, \mathrm{k})$;

We compute the 10th order Taylor coefficient of $F_{11}$ by

$>\operatorname{simplify}(\operatorname{coeftayl}(\mathrm{F}(\mathrm{x}, 11), \mathrm{x}=0,10))$;

whence we get that this coefficient is $-\frac{12352}{5775}$, i.e., it is not zero, which means that the parameters in (16) do not provide solution to the invariance equation.

In the case when $v=-w$, we have that

$$
a=-b, \quad c=\frac{7}{3} w, \quad d=\frac{5}{3} w, \quad p=\left(1+\frac{\sqrt{13}}{3}\right) w, \quad q=\left(1-\frac{\sqrt{13}}{3}\right) w,
$$

whence a similar calculation as in the previous case shows that we again do not get an additional solution to the invariance equation. 
Acknowledgement. The authors thank the anonymous referees for their help and suggestions.

\section{REFERENCES}

[1] Sz. Baják and Zs. Páles, Computer aided solution of the invariance equation for two-variable Gini means, Comput. Math. Appl. 58 (2009), 334-340.

[2] Sz. Baják and Zs. Páles, Invariance equation for generalized quasi-arithmetic means, Aequationes Math. 77 (2009), no. 1-2, 133-145.

[3] J. M. Borwein and P. B. Borwein, Pi and the AGM, John Wiley \& Sons Inc., New York, 1987, A study in analytic number theory and computational complexity. MR 89a:11134

[4] P. Burai, Extension theorem for a functional equation, J. Appl. Anal. 12 (2006), no. 2, 293-299. MR 2008d:39025

[5] P. Burai, A Matkowski-Sutô type equation, Publ. Math. Debrecen 70 (2007), no. 1-2, 233-247. MR 2007k:39042

[6] J. Błasińska-Lesk, D. Głazowska, and J. Matkowski, An invariance of the geometric mean with respect to Stolarsky mean-type mappings, Results Math. 43 (2003), no. 1-2, 42-55. MR 2004a:39042

[7] Z. Daróczy, Gy. Maksa, and Zs. Páles, Extension theorems for the Matkowski-Sutô problem, Demonstratio Math. 33 (2000), no. 3, 547-556. MR 2002a:39027

[8] Z. Daróczy and Zs. Páles, On means that are both quasi-arithmetic and conjugate arithmetic, Acta Math. Hungar. 90 (2001), no. 4, 271-282. MR 2003g:26034

[9] Z. Daróczy and Zs. Páles, Gauss-composition of means and the solution of the Matkowski-Sutô problem, Publ. Math. Debrecen 61 (2002), no. 1-2, 157-218. MR 2003j:39061

[10] J. Domsta and J. Matkowski, Invariance of the arithmetic mean with respect to special mean-type mappings, Aequationes Math. 71 (2006), no. 1-2, 70-85. MR 2007a:26051

[11] C. Gini, Di una formula compressiva delle medie, Metron 13 (1938), 3-22.

[12] D. Głazowska, W. Jarczyk, and J. Matkowski, Arithmetic mean as a linear combination of two quasiarithmetic means, Publ. Math. Debrecen 61 (2002), no. 3-4, 455-467. MR 2003h:26045

[13] J. Jarczyk, Invariance of weighted quasi-arithmetic means with continuous generators, Publ. Math. Debrecen 71 (2007), no. 3-4, 279-294. MR 2008j:26056

[14] J. Jarczyk and J. Matkowski, Invariance in the class of weighted quasi-arithmetic means, Ann. Polon. Math. 88 (2006), no. 1, 39-51. MR 2007g:26044

[15] J. Matkowski, Invariant and complementary quasi-arithmetic means, Aequationes Math. 57 (1999), no. 1, 87-107. MR 2000g:39025

[16] J. Matkowski, On invariant generalized Beckenbach-Gini means, Functional Equations - Results and Advances (Z. Daróczy and Zs. Páles, eds.), Advances in Mathematics, vol. 3, Kluwer Acad. Publ., Dordrecht, 2002, pp. 219-230. MR 2003j:39063

[17] J. Matkowski, Lagrangian mean-type mappings for which the arithmetic mean is invariant, J. Math. Anal. Appl. 309 (2005), no. 1, 15-24. MR 2006c:26051

[18] Zs. Páles, Inequalities for differences of powers, J. Math. Anal. Appl. 131 (1988), no. 1, 271-281. MR 89f:26023

[19] K. B. Stolarsky, Generalizations of the logarithmic mean, Math. Mag. 48 (1975), 87-92. MR 50 \#10186

E-mail address: bajaksz@gmail.com

Faculty of Informatics, University of Debrecen, 4010 Debrecen, Pf 12, Hungary

E-mail address: pales@science.unideb.hu

Institute of Mathematics, University of Debrecen, 4010 Debrecen, Pf 12, Hungary 